\title{
Plantas medicinais e de uso religioso comercializadas em mercados e feiras livres no Rio de Janeiro, RJ, Brasil ${ }^{1}$
}

\author{
Sheila Karla Santos de Azevedo² e Inês Machline Silva ${ }^{2,3}$
}

Recebido em 27/06/2003. Aceito em 8/08/2005.

\begin{abstract}
RESUMO - (Plantas medicinais e de uso religioso comercializadas em mercados e feiras livres no Rio de Janeiro, RJ, Brasil). Esse estudo objetivou o levantamento das espécies comercializadas em feiras livres e mercados no Rio de Janeiro, a verificação da utilização (medicinais e/ou de uso religioso) e as formas de obtenção das mesmas (cultivo e/ou extrativismo em Mata Atlântica e/ou áreas ruderais). Foram visitadas quatro feiras livres e quatro mercados e realizadas coletas de campo, na Serra do Mendanha, acompanhadas por um informante. As informações foram provenientes de observação participante e entrevistas semi-estruturadas. Foram inventariadas 127 espécies pertencentes a 58 famílias, sendo Asteraceae (17 espécies), Lamiaceae (9), Leguminosae (7), Euphorbiacaea, Lauraceae, Piperaceae e Solanaceae (5) as de maior riqueza. A maioria das espécies é utilizada para fins terapêuticos $(70,1 \%)$ e existe um relativo equilíbrio entre extrativismo $(40,4 \%)$ e cultivo $(52,8 \%)$ para estas, com poucas incluídas nos dois casos $(6,7 \%)$. Em relação às plantas utilizadas para fins religiosos, constatou-se que o extrativismo $(64,3 \%)$ sobrepõe-se ao cultivo $(32,1 \%)$, com apenas 3,6\% em ambos os casos. A coleta se dá predominantemente na Mata Atlântica (83,3\%), em áreas que fazem parte ou circundam Unidades de Conservação. Isto reflete a crença de que estas espécies devam ser retiradas preferencialmente de seus locais de origem. Sugere-se que a pressão de coleta exercida sobre as plantas de uso religioso na Mata Atlântica contribua para a vulnerabilidade das mesmas.
\end{abstract}

Palavras-chave : extrativismo, Mata Atlântica, etnobotânica, Rio de Janeiro

\begin{abstract}
Medical and religious plants commercialized in conventional and open-air markets of Rio de Janeiro municipality Rio de Janeiro State, Brazil). This paper has as a main goal to study the species commercialized in both conventional and open-air markets in Rio de Janeiro. It is reported the concurrency of the type of use (medical and/or religious) together with the collection procedure of these plants, arising from cultivation and/or extraction from the Atlantic Rain Forest and/or from wastelands. Four open-air markets and four conventional markets were visited for this study, while the field collection in the Serra do Mendanha was accompanied by an informant. Data collection was obtained through participant observation and semi structured interviews. Most of the species are used for therapeutical purpose (70.1\%), observing that there is a certain balance between plants colletcted (40.4\%) and cultivated (52.8\%), and the remaining $(6.7 \%)$, including both. Regarding the plants used for religious purpose it was observed that explotation from nature $(64.3 \%)$ ovelaps cultivation (32.1\%), with only 3.6\% yields from both cases. Most of the collects occurs within conservation areas of the Atlantic Rain Forest (83.3\%). This result reflects the popular belief that these species need to be extracted from its native area. The vulnerability of these species may well be related to this practice.
\end{abstract}

Key words: extrativism, Atlantic Rain Forest, ethnobotany, Rio de Janeiro

\section{Introdução}

A desarticulação de políticas públicas relativas ao atendimento das necessidades básicas de saúde das populações periféricas vem levando a uma crescente procura de alternativas economicamente mais viáveis, o que gera um aumento do consumo de plantas medicinais. $\mathrm{O}$ atual valor medicinal de muitas espécies vegetais deve-se em parte à divulgação das vantagens da fitoterapia e, principalmente, à constante elevação de preços dos medicamentos industrializados. Neste sentido, Montanari Junior (2002) assinala que, como conseqüência da revalorização mundial do uso de plantas medicinais, a pressão ecológica exercida sobre alguns desses recursos naturais tem sido grande nos últimos anos. Acrescenta ainda que o valor econômico dessas plantas põe em perigo a sobrevivência de muitas espécies medicinais nativas. Sánchez \& Valverde (2000) assinalam que o comércio local de plantas medicinais leva à deterioração de populações naturais, tanto quanto a pressão extrativista da indústria de fitofármacos.

\footnotetext{
1 Parte da Monografia de Bacharelado da primeira Autora. Bolisita FAPESP

2 Universidade Federal Rural do Rio de Janeiro, Departamento de Botânica, Rodovia BR 465 Km 7, CEP 23890-000, Seropédica, RJ, Brasil

3 Autor para correspondência: machline@ufrrj.br
} 
De acordo com a Organização Mundial de Saúde, $80 \%$ das pessoas dos países em desenvolvimento no mundo dependem da medicina tradicional para as suas necessidades básicas de saúde e cerca de $85 \%$ da medicina tradicional envolve o uso de extratos de plantas. Isso significa que 3,5 a 4,0 bilhões de pessoas dependem de plantas como fontes de drogas (Farnsworth 1997). O aumento no uso de plantas medicinais está provavelmente relacionado à deterioração das condições econômicas nos países do terceiro mundo (Hersch-Martínez 1995).

Estudos efetuados na África vêm apontando para a virtual extinção de muitas espécies em função do excesso de coletas decorrentes da demanda urbana atual pela utilização de plantas medicinais, reforçando a necessidade de se apurar os impactos, em longo prazo, da ação das populações que utilizam a flora local (Williams et al. 2000). Cunningham (1998) afirma que entre os recursos vegetais silvestres utilizados na zona rural, as plantas medicinas estão entre aqueles que, apesar da urbanização em muitas cidades na África, continuam sendo muito utilizados. As cidades são um potente foco de demanda que se alimentam das zonas rurais, exercendo assim pressão sobre os recursos naturais silvestres.

O Brasil é um dos países de maior diversidade genética vegetal, contando com mais de 55.000 espécies catalogadas (Nodari \& Guerra 1999). Neste contexto, a Mata Atlântica ocupa posição de destaque no que se refere ao patrimônio genético nacional. Sua ocupação deu-se ao longo dos 500 anos de história recente por diferentes formas de exploração, o que levou à depleção e extinção de muitos recursos. PavanFruehauf (2000) lembra que o extrativismo de produtos vegetais, como a exploração do pau-brasil, é que deu início à colonização e passou de forma intensiva a muitos outros recursos naturais. Lima et al. (2002) afirmam que a Mata Atlântica, mais do que a Floresta Amazônica,é o palco da maior tragédia ecológica verificada no Brasil.

No Brasil, a população rural sempre fez uso de plantas medicinais, mas com a expansão de seu uso entre a população urbana, iniciou-se uma pressão extrativista nos locais onde ainda se pode encontrar populações de espécies com valor de mercado. Por seu baixo custo, as plantas medicinais representam, em muitos casos, a única alternativa possível para esta parcela da população. Acrescenta-se o fato de que, assim como foi assinalado para a África do sul por Huntley et al. (apud Williams et al. 2000), a atividade econômica dos erveiros pode se tornar uma atividade rentável, onde taxas formais de desemprego sejam altas.

No município do Rio de Janeiro, as pressões antrópicas vêm impactando de forma alarmante os últimos remanescentes de floresta que ainda encontram-se protegidos em Unidades de Conservação. Desde o início do processo de colonização, o município perdeu aproximadamente $96 \mathrm{~km}^{2}$ de florestas, manguezais e outros ambientes naturais (César \& Oliveira 1992). Além da ocupação indevida de encostas, dos incêndios florestais e de vetores difusos de desflorestamento, como a deposição de poluentes, muitas espécies estão sendo retiradas destas áreas para serem vendidas como medicinais e/ou para serem utilizadas em rituais religiosos.

Procurou-se neste trabalho elaborar um levantamento das plantas comercializadas em feiras e mercados, fornecendo informações acerca da procedência das espécies, aspectos etnobotânicos e de comercialização de plantas medicinais e de uso religioso em feiras livres e mercados de cinco bairros do município do Rio de Janeiro, com enfoque naquelas espécies retiradas da Mata Atlântica.

\section{Material e métodos}

As informações acerca da coleta e comercialização foram obtidas de janeiro a dezembro/1999, em quatro feiras livres e quatro mercados do município do Rio de Janeiro, localizados nos bairros de Campo Grande, Realengo, Taquara, Irajá e Madureira. Estes bairros circundam alguns dos principais maciços montanhosos do Rio de Janeiro (Fig. 1). Durante este período foram feitas visitas periódicas às feiras livres e aos mercados. O mercado de Madureira e a feira de Campo Grande foram visitados quinzenalmente, nos meses de janeiro a junho e abril a dezembro, respectivamente. O primeiro é o maior mercado dentre os trabalhados. Na ocasião da pesquisa era constituído por cerca de 700 lojas para venda de muitos produtos. O setor conhecido como o "pavilhão das ervas" apresentava 29 boxes (bancas) destinados à venda de hortaliças, temperos e plantas utilizadas como medicinais e/ou nos cultos afro-brasileiros. Na Feira de Campo Grande - a mais expressiva em plantas medicinais dentre aquelas utilizadas na pesquisa - um de seus erveiros tornou-se informante em expedições para coleta e observação de plantas na natureza.

Foram realizados expedições à Serrinha, na Serra do Mendanha, para coleta e observação de material botânico, em caminhadas livres (walk-in-the-woods) 


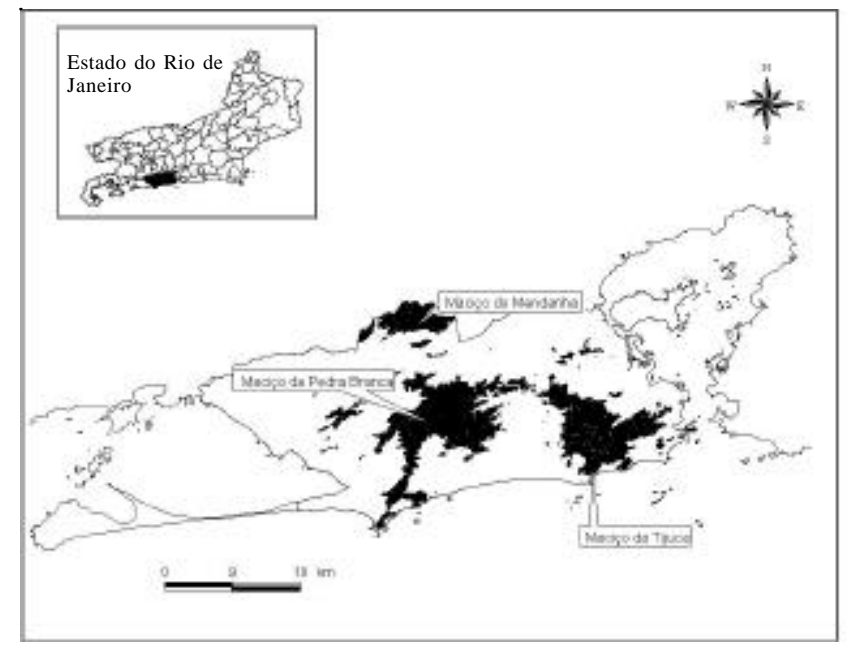

Figura 1. Mapa do município do Rio de Janeiro e das principais serranias (fonte: Laboratório de Geotecnologias PUC-Rio).

(Alexiades 1966) acompanhados pelo informante supra citado. A escolha das trilhas e a seleção das plantas foram indicadas pelo informante que se auto denominava mateiro e conhecedor da vegetação local. Durante as caminhadas foram anotados dados referentes aos nomes e usos das plantas.

Apesar de no Rio de Janeiro utilizar-se com muita frequiência o termo erveiro como sinônimo de mateiro optou-se, para melhor entendimento do texto, categorizá-los distintamente: erveiro é aquele que mantém uma banca para comercialização de plantas; mateiro, aquele que extrai as plantas em ambientes antropicamente alterados ou não. De modo geral estes últimos são apontados como conhecedores de plantas e da mata.

As informações sobre a coleta e comercialização das plantas foram provenientes de observação participante, conversas informais e de entrevistas semiestruturadas (Martin 1995) com os feirantes (erveiros) e de conversas informais com mateiros. As espécies estudadas foram obtidas nas feiras livres e mercados e por coleta na serra do Mendanha. Os materiais botânicos obtidos foram herborizados, identificados e incorporados ao acervo do Herbário do Departamento de Botânica da Universidade Federal Rural do Rio de Janeiro (RBR). A identificação botânica foi feita através da utilização de chaves analíticas, por comparação nos herbários do Departamento de Botânica da UFRRJ (RBR) e do Instituto de Pesquisas Jardim Botânico do Rio de Janeiro (RB) ou com auxílio de especialistas.

Elaborou-se uma listagem de espécies organizada por ordem alfabética de famílias botânicas seguidas pelos nomes científicos, nomes populares, indicação popular de uso e formas de obtenção das plantas. A indicação popular foi categorizada em "medicinal" e/ou "religiosa" e as formas de obtenção divididas em "cultivo" e/ou "extrativismo". Como forma de se simplificar o que os informantes consideram "de dentro" ou "de fora" da mata, optou-se por dividir o extrativismo ocorrendo em "Mata Atlântica" e/ou "áreas ruderais" (como terrenos baldios e ruas).

\section{Resultados e discussão}

O comércio das plantas utilizadas como medicinais e de uso religioso no município do Rio de Janeiro pode ser caracterizado pelo mercado formal, que inclui os feirantes (erveiros) com licença para utilização de bancas em feiras ou mercados e pelo mercado informal, constituído por vendedores não legalizados que comercializam as plantas em bancas isoladas e nas calçadas, geralmente próximas às feiras livres e mercados, mas não fazendo parte dos mesmos.

A CEASA/RJ (Centrais de Abastecimento do Estado do Rio de Janeiro S.A.) em Irajá, juntamente com o Mercado de Madureira constituem os dois principais centros de distribuição de plantas para as feiras e pequenos mercados.

Os temperos e outras espécies amplamente utilizadas como medicinais pela população como manjericão (Ocimum americanum L.), alfavaca (Ocimum gratissimum L.), alecrim (Rosmarinus officinalis L.), poejo (Mentha pulegium L.) e carqueja (Baccharis trimera (Less.) DC.), comercializadas pelos feirantes destes dois mercados, têm diferentes procedências. Podem vir do cultivo de suas próprias hortas e quintais ou então compradas de produtores especializados que as cultivam.

As espécies típicas de ambientes muito alterados tais como Schinus terebinthifolius Raddi (aroeira), Chenopodium ambrosioides L. (mastruço), Vernonia polyanthes Less. (assa-peixe) e outras, são retiradas destes ambientes pelos próprios feirantes ou então por mateiros que também são responsáveis pela coleta de espécies de vegetação mais densa, em sucessão secundária.

A CEASA está vinculado à Secretaria de Agricultura, Abastecimento, Pesca e Desenvolvimento do estado do Rio de Janeiro. Tem como objetivos básicos promover, desenvolver, dinamizar e organizar a comercialização de produtos hortigranjeiros, ao nível de atacado. No pavilhão destinado às verduras, encontram-se as espécies de uso medicinal e religioso, 
que são comercializadas, em grande parte, durante a madrugada, para erveiros de feiras livres, pequenos mercados e bancas isoladas. $\mathrm{O}$ contato entre os erveiros e mateiros costuma ser bastante rápido, sendo suficiente para que o pagamento e pedido de novas encomendas, ocorram.

O Mercado de Madureira, fundado no início do século passado, servia como centro de distribuição de alimentos para a zona suburbana da cidade do Rio de Janeiro. Tornou-se, nas últimas décadas, um mercado tipicamente popular, com vendas a atacado e varejo, que funciona todos os dias da semana, exceto aos domingos, no período de $7 \mathrm{~h}$ às $19 \mathrm{~h}$. Inclui diversas papelarias, drogarias, mercearias e lojas especializadas, principalmente, no comércio de artigos religiosos. Para Falcão (2002) este mercado é uma via para se conhecer o mundo afro-brasileiro e apresenta-se como mediador de um processo de socialização das diversas categorias de pessoas iniciadas no candomblé, funcionando como uma caixa de ressonância dos terreiros. $\mathrm{Na}$ área denominada de pavilhão das ervas, as plantas são trazidas pelos mateiros, geralmente na hora de abertura do mercado, dentro de sacos de aniagem ou similar. Os ramos de cada espécie são agrupados em molhos grandes (também chamados de "amarrados") que são dispostos no chão do corredor central, entre os boxes de venda, para que sejam analisados e comprados pelos feirantes (erveiros) que, por sua vez, os subdividem em três a quatro molhos menores, para serem revendidos aos consumidores ou então a feirantes de outras feiras que, por sua vez, irão fragmentar os molhos, para posterior revenda.

A cadeia de comercialização das espécies estudadas encontra-se ilustrada na Fig. 2.

Usos e origem das espécies comercializadas - Foram encontradas 127 espécies comercializadas, pertencentes a 58 famílias botânicas, sendo as mais representativas Asteraceae (17 espécies); Lamiaceae (nove espécies).

Leguminosae (sete espécies); Euphorbiaceae; Lauraceae; Piperaceae e Solanaceae (cinco espécies) (Tab. 1). As famílias Asteraceae, Lamiaceae e Solanaceae também foram as mais representativas nos estudos realizados por Parente \& Rosa (2001), no Município de Barra do Piraí (RJ). Almeida \& Albuquerque (2002) encontraram, na feira de Caruaru (PE), um total de 114 espécies comercializadas, inseridas em 57 famílias, sendo Lamiaceae, Leguminosae, Euphorbiaceae, Lauraceae, Asteraceae e Bignoniaceae, as famílias com maior número de espécies. Bennet \& Prance (2000) também apontaram

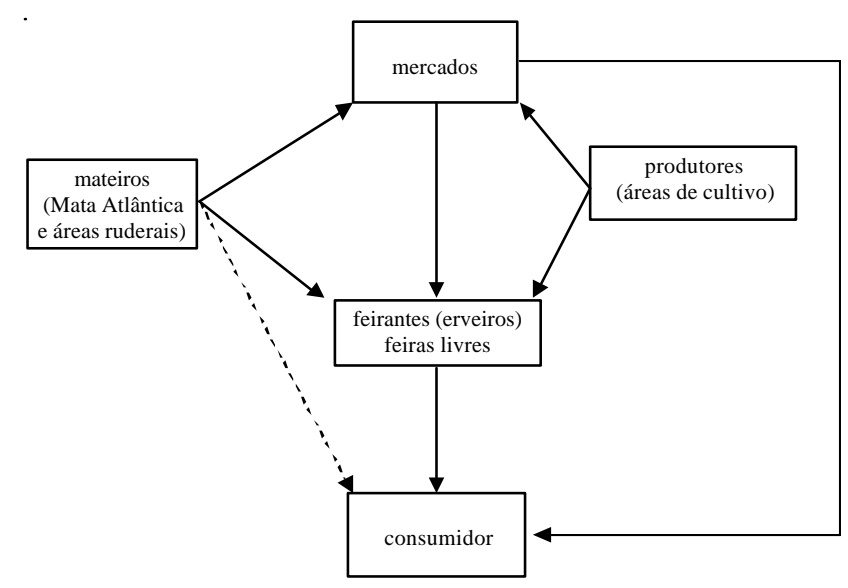

Figura 2. Cadeia de comercialização das espécies de uso medicinal e/ou religioso no município do Rio de Janeiro, RJ, Brasil. A linha tracejada indica fluxo menos freqüente.

as famílias Asteraceae, Lamiaceae e Leguminosae como culturalmente importantes, por serem amplamente distribuídas em regiões temperadas e tropicais do mundo. Por outro lado, são famílias com muitas espécies herbáceas ou arbustivas ruderais e/ou já domesticadas e de fácil cultivo.

Quanto à indicação popular, foi constatado que a maioria das espécies é utilizada para fins medicinais $(70,1 \%)$, seguidas daquelas usadas para fins religiosos $(22,0 \%)$. Segundo informações dos feirantes, estas últimas são muito procuradas por serem indicadas por dirigentes de cultos afro-brasileiros. Para Falcão (2002), o Mercado de Madureira reúne tudo de necessário para a vida ritual em um terreiro de candomblé e os vendedores de ervas formam um grupo de vital importância para os cultos. Há ainda as que são consideradas nas duas categorias $(7,9 \%)$.

Os feirantes informaram que muitas espécies passam a ser consideradas medicinais à medida que "entram em moda", por aparecerem nos meios de comunicação de massa, tornando-se então muito procuradas. Neste sentido, Amorozo (2002) também identificou nas comunidades de agricultores estudadas em Mato Grosso, a entrada de novas informações com relação à utilização de plantas para fins medicinais através, principalmente, da televisão.

Considerando-se todas as espécies comercializadas, observa-se um equilíbrio entre o cultivo e o extrativismo (Fig. 3), sendo que este último ocorre predominantemente em ambientes de Mata Atlântica (Fig. 4).

Em relação às espécies utilizadas como medicinais, o cultivo foi apontado para a maioria delas $(52,8 \%)$, seguido do extrativismo $(40,4 \%)$ ou ambos $(6,7 \%)$. A 
Tabela 1. Lista de espécies de plantas medicinais e de uso religioso comercializadas em mercados e feiras livres no município do Rio de Janeiro, RJ, Brasil. (m.a. = mata atlântica; a.r. = áreas ruderais).

\begin{tabular}{|c|c|c|c|}
\hline Família/Táxon & Nome popular & Indicação & Forma de obtenção \\
\hline \multicolumn{4}{|l|}{ ACANTHACEAE } \\
\hline $\begin{array}{l}\text { Justicia pectoralis Jacq. } \\
\text { AGAVACEAE }\end{array}$ & anador & medicinal & cultivo \\
\hline Agave americana $\mathrm{L}$. & pita & medicinal & cultivo \\
\hline Sansevieria trifasciata Hort. ex Prain & espada-de-são- jorge & religioso & cultivo \\
\hline \multicolumn{4}{|l|}{ ALISMATACEAE } \\
\hline Echinodorus grandiflorus Mich. & chapéu-de-couro & medicinal & cultivo/extrativismo (a.r.) \\
\hline \multicolumn{4}{|l|}{ ANACARDIACEAE } \\
\hline Mangifera indica $\mathrm{L}$. & mangueira & medicinal & cultivo \\
\hline Schinus terebinthifolius Raddi & aroeira & medicinal/religioso & extrativismo (a.r.) \\
\hline \multicolumn{4}{|l|}{ ANNONACEAE } \\
\hline \multicolumn{2}{|l|}{ APIACEAE } & medicinal & cultivo \\
\hline Foeniculum vulgare Mill. & erva-doce & medicinal & cultivo \\
\hline \multicolumn{4}{|l|}{ APOCYNACEAE } \\
\hline Geissospermum laeve (Vell.) Miers & pau-pereira & medicinal & extrativismo (m.a.) \\
\hline \multicolumn{4}{|l|}{ ARACEAE } \\
\hline Pistia stratiotes L. & erva-de-santa luzia & medicinal & cultivo \\
\hline \multicolumn{4}{|l|}{ ARECACEAE } \\
\hline Elaeis guineensis Jacq. & dendezeiro & religioso & cultivo \\
\hline \multicolumn{4}{|l|}{ ARISTOLOCHIACEAE } \\
\hline Aristolochia aff. trilobata $\mathrm{L}$. & mil-homens, mal-com-tudo & medicinal/religioso & extrativismo (m.a.) \\
\hline \multicolumn{4}{|l|}{ ASCLEPIADACEAE } \\
\hline Asclepias curassavica $\mathrm{L}$. & oficial-de-sala & medicinal & extrativismo (m.a.) \\
\hline \multicolumn{4}{|l|}{ ASTERACEAE } \\
\hline Achyrocline satureoides (Lam.) DC. & macela & medicinal & extrativismo (a.r.) \\
\hline Ageratum conyzoides $\mathrm{L}$. & erva-de-são joão & medicinal & extrativismo (a.r.) \\
\hline Artemisia absinthium $\mathrm{L}$. & losna & medicinal & cultivo \\
\hline Baccharis dracunculifolia DC. & alecrim-do- campo & medicinal/religioso & extrativismo (a.r.) \\
\hline B. trimera (Less.) DC. & carqueja & medicinal & extrativismo (m.a. e a.r.) \\
\hline Bidens pilosa $\mathrm{L}$. & picão & medicinal & extrativismo (a.r.) \\
\hline Coreopsis grandiflora $\mathrm{L}$. & camomila & medicinal & cultivo \\
\hline Elephantopus scaber L. & língua-de-vaca, erva-grossa & medicinal & extrativismo (a.r.) \\
\hline Emilia sonchifolia DC. & serralha & medicinal & extrativismo (a.r.) \\
\hline Matricaria recutita $\mathrm{L}$. & camomila & medicinal & cultivo \\
\hline Mikania glomerata Spreng. & guaco & medicinal & cultivo \\
\hline M. hirsutissima DC. & cipó-cabeludo & medicinal & extrativismo (m.a.) \\
\hline Mikania sp. & $\begin{array}{l}\text { mal-com-deus, } \\
\text { bem-com-tudo }\end{array}$ & religioso & extrativismo (m.a.) \\
\hline Pluchea sagittalis (Cham.) Cabrera & quitoco & medicinal & cultivo/extrativismo (a.r.) \\
\hline Solidago chilensis Meyen & arnica-do-campo & medicinal & cultivo \\
\hline Stevia rebaudiana (Bertoni) Bertoni & estévia & medicinal & cultivo \\
\hline Vernonia polyanthes Less. & assa-peixe & medicinal & extrativismo (a.r.) \\
\hline \multicolumn{4}{|l|}{ BIGNONIACEAE } \\
\hline Cybistax antisyphilitica (Mart.) Mart. & cinco-em-folhas & medicinal & extrativismo (m.a.) \\
\hline Jacaranda puberula Cham. & carobinha & medicinal & extrativismo (m.a.) \\
\hline Tynanthus labiatus (Cham.) Miers & cipó-cravo & medicinal & extrativismo (m.a.) \\
\hline \multicolumn{4}{|l|}{ BIXACEAE } \\
\hline Bixa orellana $\mathrm{L}$. & urucum & religioso & cultivo \\
\hline \multicolumn{4}{|l|}{ BROMELIACEAE } \\
\hline Tillandsia usneoides L. & barba-de-velho & religioso & extrativismo (m.a.) \\
\hline \multicolumn{4}{|l|}{ CACTACEAE } \\
\hline Epiphyllum phyllanthus (L.) Haw. & dama-da-noite & religioso & extrativismo (m.a.) \\
\hline \multicolumn{4}{|l|}{ CAPRIFOLIACEAE } \\
\hline Sambucus australis Cham. \& Schlecht. & sabugueiro & medicinal & cultivo \\
\hline
\end{tabular}


Tabela 1 (continuação)

\begin{tabular}{|c|c|c|c|}
\hline Família/Táxon & Nome popular & Indicação & Forma de obtenção \\
\hline \multicolumn{4}{|l|}{ CECROPIACEAE } \\
\hline Cecropia glaziovi Snethl. & embaúba & medicinal & extrativismo (m.a.) \\
\hline \multicolumn{4}{|l|}{ CHENOPODIACEAE } \\
\hline \multicolumn{3}{|l|}{ CRASSULACEAE } & cultivo/extrativismo (a.r.) \\
\hline Kalanchoe brasiliensis Camb. & saião & medicinal & cultivo \\
\hline \multicolumn{4}{|l|}{ CUCURBITACEAE } \\
\hline Luffa operculata (L.) Cogn. & buchinha & medicinal & cultivo \\
\hline Mormodica charantia $\mathrm{L}$. & melão-de-são caetano & medicinal & cultivo/extrativismo (a.r.) \\
\hline \multicolumn{4}{|l|}{ CUSCUTACEAE } \\
\hline Cuscuta sp. & cipó-chumbo & medicinal & extrativismo (a.r.) \\
\hline \multicolumn{4}{|l|}{ CYPERACEAE } \\
\hline Scleria pterota Presl. & navalha-de-macaco & medicinal & extrativismo (a.r.) \\
\hline \multicolumn{4}{|l|}{ DILLENIACEAE } \\
\hline Davilla rugosa Poir. & cipó-cabloco & religioso & extrativismo (m.a.) \\
\hline \multicolumn{4}{|l|}{ EQUISETACEAE } \\
\hline Equisetum arvense $\mathrm{L}$. & cavalinha & medicinal & cultivo \\
\hline \multicolumn{4}{|l|}{ EUPHORBIACEAE } \\
\hline Acalypha poiretii Spreng. & parietaria & medicinal & cultivo/extrativismo (a.r.) \\
\hline Jatropha gossypiifolia L. & pinhão-roxo & religioso & cultivo \\
\hline Joannesia princeps Vell. & cutieira, boleira & medicinal & extrativismo (a.r. e m.a.) \\
\hline Phyllanthus niruri $\mathrm{L}$. & quebra-pedra & medicinal & cultivo \\
\hline Ricinus communis $\mathrm{L}$. & mamona & religioso & cultivo/extrativismo (a.r.) \\
\hline \multicolumn{4}{|l|}{ FLACOURTIACEAE } \\
\hline Casearia sylvestris $\mathrm{Sw}$. & são gonçalinho & religioso & extrativismo m.a \\
\hline \multicolumn{4}{|l|}{ LAMIACEAE } \\
\hline Leonurus sibiricus L. & erva-macaé & medicinal & extrativismo (a.r.) \\
\hline Leonotis nepetaefolia (L.) R. Br. & cordão-de-frade & religioso & extrativismo (a.r.) \\
\hline Mentha piperita var. citrata (Ehrh.) Briq. & alevante, elevante & medicinal & cultivo \\
\hline M. pulegium $\mathrm{L}$. & poejo & medicinal & cultivo \\
\hline Ocimum americanum $\mathrm{L}$. & manjericão roxo & medicinal/religioso & cultivo \\
\hline O. gratissimum $\mathrm{L}$. & alfavaca & medicinal & cultivo \\
\hline O. sellowii Benth. & anis, elixir-paregórico & medicinal & cultivo \\
\hline Plectranthus barbatus Andr. & boldo & medicinal & cultivo \\
\hline Rosmarinus officinalis L. & alecrim & medicinal/religioso & cultivo \\
\hline \multicolumn{4}{|l|}{ LAURACEAE } \\
\hline $\begin{array}{l}\text { Cinnamomum aff. triplinerve } \\
\text { (Ruiz \& Pav.) Kosterm. }\end{array}$ & canela & medicinal & extrativismo (m.a.) \\
\hline Laurus nobilis $\mathrm{L}$. & louro & medicinal & cultivo \\
\hline Ocotea glaziovii Mez. & canela preta & medicinal & extrativismo (m.a.) \\
\hline O. odorifera (Vell.) Rohwer & canela-sassafrás & medicinal & extrativismo (m.a.) \\
\hline Persea americana Mill. & abacate & medicinal & cultivo \\
\hline \multicolumn{4}{|l|}{ LEGUMINOSAE } \\
\hline Bauhinia forficata Link & pata-de-vaca & medicinal & extrativismo (m.a.) \\
\hline B. microstachya (Raddi) Macbr. & pata-de-vaca & medicinal & extrativismo (m.a.) \\
\hline B. variegata $\mathrm{L}$. & pata-de-vaca & medicinal & cultivo \\
\hline Bowdichia sp. & batata-de-sucupira & medicinal & extrativismo (m.a.) \\
\hline Cajanus cajan (L.) Mill. & feijão-guandu & medicinal & cultivo \\
\hline Caesalpinia ferrea Mart. & jucá, fruto-de-jucá & religioso & cultivo \\
\hline Senna occidentalis (L.) Link & fedegoso & medicinal & extrativismo (m.a.) \\
\hline \multicolumn{4}{|l|}{ LILIACEAE } \\
\hline Allium cepa $\mathrm{L}$. & cebola & medicinal & cultivo \\
\hline Aloe vera (L.) Burm. f. & babosa & medicinal & cultivo \\
\hline Herreria salsaparrilha Mart. & salsaparrilha & medicinal & cultivo \\
\hline \multicolumn{4}{|l|}{ LYTHRACEAE } \\
\hline Cuphea carthagenensis (Jacq.) Macbr. & sete-sangrias & medicinal & extrativismo (a.r.) \\
\hline \multicolumn{4}{|l|}{ MALVACEAE } \\
\hline Gossypium sp. & algodão & medicinal & cultivo \\
\hline
\end{tabular}


Tabela 1 (continuação)

\begin{tabular}{|c|c|c|c|}
\hline Família/Táxon & Nome popular & Indicação & Forma de obtenção \\
\hline \multicolumn{4}{|l|}{ MELASTOMATACEAE } \\
\hline Miconia albicans (Sw.) Triana & canela-de-velho & religioso & extrativismo (m.a.) \\
\hline M. calvescens Schrank \& Mart. & abranda-fogo, erva-de-xangô & religioso & extrativismo (m.a.) \\
\hline \multicolumn{4}{|l|}{ MELIACEAE } \\
\hline Melia azedarach L. & para-raio & religioso & cultivo \\
\hline \multicolumn{4}{|l|}{ MONIMIACEAE } \\
\hline Mollinedia schottiana (Spreng.) Perkins & quebra-mironga & religioso & extrativismo (m.a.) \\
\hline Siparuna guianensis Aublet & negramina & religioso & extrativismo (m.a.) \\
\hline \multicolumn{4}{|l|}{ MORACEAE } \\
\hline Artocarpus incisus (Thunb.) L.f. & fruta-pão & medicinal & cultivo \\
\hline Dorstenia sp. & carapiá & medicinal & extrativismo (m.a.) \\
\hline Sorocea guilleminiana Gaudich. & espinheira-santa & medicinal & extrativismo (m.a.) \\
\hline \multicolumn{4}{|l|}{ MUSACEAE } \\
\hline Musa paradisiaca $\mathrm{L}$. & bananeira & medicinal & cultivo \\
\hline \multicolumn{4}{|l|}{ MYRTACEAE } \\
\hline Eucalyptus sp. & eucalipto & medicinal & cultivo \\
\hline Eugenia jambolana Lam. & jambolão & medicinal & cultivo \\
\hline E. uniflora $\mathrm{L}$. & pitanga & medicinal & cultivo/extrativismo (m.a.) \\
\hline \multicolumn{4}{|l|}{ NYMPHEACEAE } \\
\hline Nymphaea sp. & chibatada & religioso & cultivo \\
\hline \multicolumn{4}{|l|}{ OXALIDACEAE } \\
\hline Averrhoa carambola $\mathrm{L}$. & carambola & medicinal & cultivo \\
\hline \multicolumn{4}{|l|}{ PASSIFLORACEAE } \\
\hline Passiflora edulis Sims & maracujá & medicinal & cultivo \\
\hline \multicolumn{4}{|l|}{ PHYTOLACCACEAE } \\
\hline Petiveria alliacea $\mathrm{L}$. & guiné piu-piu & medicinal/religioso & extrativismo (a.r.) \\
\hline Gallesia integrifolia (Spreng.) Harms & pau-d'alho & medicinal & extrativismo (m.a.) \\
\hline \multicolumn{4}{|l|}{ PIPERACEAE } \\
\hline Ottonia sp. & desmancha-nó & religioso & extrativismo (m.a.) \\
\hline Ottonia anisum Spreng. & joão-barandí & medicinal & extrativismo (m.a.) \\
\hline Piper arboreum Aubl. & vence-demanda & religioso & extrativismo (m.a.) \\
\hline P. umbellatum L. & capeba & religioso & extrativismo (m.a.) \\
\hline Piper sp. & quebra-mandinga & religioso & extrativismo (m.a.) \\
\hline \multicolumn{4}{|l|}{ PLANTAGINACEAE } \\
\hline Plantago major L. & tanchagem & medicinal & cultivo \\
\hline \multicolumn{4}{|l|}{ POACEAE } \\
\hline Coix lacryma-jobi $\mathrm{L}$. & lágrima-de-nossa-senhora & medicinal & cultivo \\
\hline Cymbopogon citratos (DC.) Staff. & capim-limão & medicinal & cultivo \\
\hline Eleusine indica (L.) Gaertn. & capim-pé-de galinha & religioso & extrativismo (a.r.) \\
\hline \multicolumn{4}{|l|}{ POLYGONACEAE } \\
\hline Polygonum hydropiperoides Michx. & erva-de-bicho & medicinal & extrativismo (a.r.) \\
\hline \multicolumn{4}{|l|}{ PUNICACEAE } \\
\hline Punica granatum L. & romã & medicinal & cultivo \\
\hline \multicolumn{4}{|l|}{ ROSACEAE } \\
\hline Rosa aff. centifolia L. & rosa-branca miúda & medicinal & cultivo \\
\hline \multicolumn{4}{|l|}{ RUBIACEAE } \\
\hline Coffea arabica $\mathrm{L}$. & café & medicinal & cultivo \\
\hline Genipa americana $\mathrm{L}$. & genipapo & medicinal & cultivo \\
\hline RUTACEAE & & & \\
\hline Citrus aurantium $\mathrm{L}$. & laranja-da-terra & medicinal/religioso & cultivo \\
\hline Ruta graveolens $\mathrm{L}$. & arruda & religioso & cultivo \\
\hline Zanthoxylum rhoifolium Lam. & espinho-cheiroso & religioso & extrativismo (m.a.) \\
\hline SCHIZEACEAE & & & \\
\hline Lygodium volubile Sw. & abre-caminho & religioso & extrativismo (m.a.) \\
\hline SOLANACEAE & & & \\
\hline Solanum aculeatissimum Jacq. & arrebenta-cavalo & religioso & extrativismo (a.r.) \\
\hline S. argenteum Dun. ex Poir. & erva-prata, santa-bárbara & medicinal/religioso & extrativismo (a.r.) \\
\hline S. americanum Mill. & maria-pretinha & medicinal & extrativismo (a.r.) \\
\hline S. cernuum Vell. & panacéia & medicinal/religioso & extrativismo (a.r.) \\
\hline S. paniculatum L. & jurubeba & medicinal & extrativismo (a.r.) \\
\hline
\end{tabular}


Tabela 1 (continuação)

\begin{tabular}{|c|c|c|c|}
\hline Família/Táxon & Nome popular & Indicação & Forma de obtenção \\
\hline \multicolumn{4}{|l|}{ URTICACEAE } \\
\hline Pilea sp. & dinheiro-em-penca & religioso & cultivo \\
\hline \multicolumn{4}{|l|}{ VERBENACEAE } \\
\hline Lantana lilacina Desf. & cambará-roxo & medicinal & extrativismo (a.r.) \\
\hline Lippia alba (Mill.) N.E. Br. & erva-cidreira & medicinal & cultivo \\
\hline Stachytarpheta cayennensis (Rich.) Vahl & gervão-roxo & medicinal & extrativismo (a.r.) \\
\hline \multicolumn{4}{|l|}{ VIOLACEAE } \\
\hline Anchietea pyrifolia A. St.-Hil. & suma-roxa & medicinal & extrativismo (m.a.) \\
\hline \multicolumn{4}{|l|}{ VITACEAE } \\
\hline Vitis cissoides (Blume) Backer & insulina vegetal & medicinal & cultivo \\
\hline \multicolumn{4}{|l|}{ ZINGIBERACEAE } \\
\hline Alpinia zerumbet (Pers.) Burtt \& Smith & colônia & medicinal/religioso & cultivo \\
\hline Costus spiralis (Jacq.) Roscoe & cana-do-brejo & medicinal & cultivo \\
\hline Zingiber officinale Roscoe & gengibre & medicinal & cultivo \\
\hline
\end{tabular}

coleta de material se dá em remanescentes de Mata Atlântica $(52,8 \%)$, em áreas ruderais $(41,7 \%)$ ou nos dois ambientes (5,6\%). Amorozo (2002) registrou 56\% das espécies medicinais crescendo espontaneamente em ambientes naturais ou antropicamente modificados, $41 \%$ cultivadas e somente $3 \%$ adquiridos por compra.

As espécies de uso religioso são provenientes na maioria do extrativismo $(64,3 \%)$, seguidas do cultivo $(32,1 \%)$ com $36 \%$ encontrando-se em ambos os casos. Este extrativismo se dá predominantemente na Mata Atlântica $(83,3 \%)$ em relação àquelas retiradas de áreas ruderais $(16,7 \%)$, o que provavelmente reflete a crença de que estas espécies devam ser retiradas prioritariamente de seu local de origem. De acordo com Trindade et al. (2000), os vegetais cultivados têm um emprego sacro no candomblé, entretanto afirmam

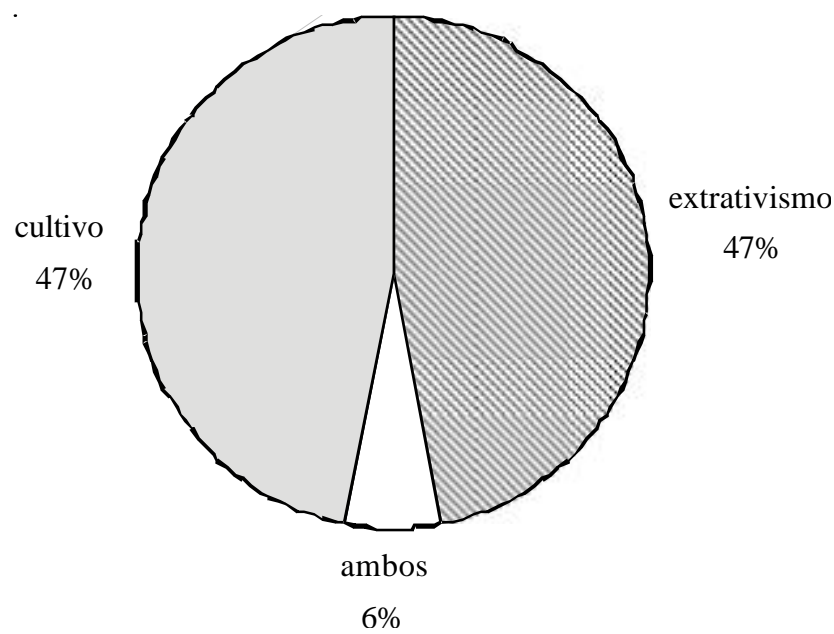

Figura 3. Formas de obtenção das espécies comercializadas para uso medicinal e/ou religioso no município do Rio de Janeiro, RJ, Brasil. que a utilização de vegetais colhidos em área não cultivada é indispensável ao culto religioso. Camargo (1998) chama a atenção para a influência portuguesa e indígena ao acervo de plantas empregadas em rituais afro-brasileiros e lembra que, na medida em que os negros foram se fixando em novas terras, desprovidos dos recursos naturais de que dispunham em suas regiões de origem, encontraram não só plantas conhecidas, como foram também se aproximando de sucedâneos. Da mesma forma Ugent (2000) relata a utilização de numerosas espécies asiáticas e européias por curandeiros no México.

Verificou-se que a Mata Atlântica pode ser considerada como um bioma fornecedor de grande número de espécies, tanto para uso medicinal quanto religioso. As informações obtidas nas entrevistas com

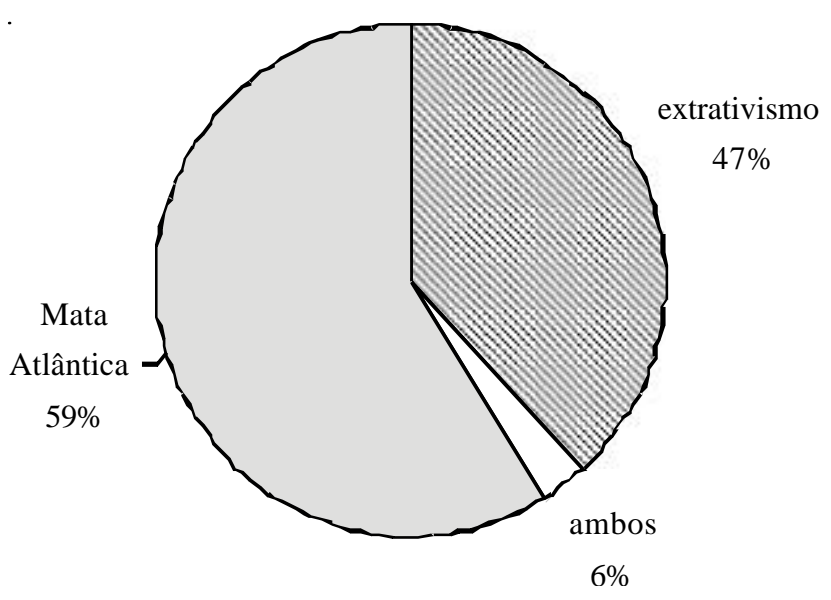

Figura 4. Locais de coleta das espécies comercializadas para uso medicinal e/ou religioso no município do Rio de Janeiro, RJ, Brasil. 
mateiros e feirantes demonstram que a grande parte das plantas comercializadas é coletada nas serranias do município do Rio de Janeiro (Serra do Mendanha e maciços da Pedra Branca e da Tijuca), bem como em municípios vizinhos como Barra do Piraí, Guapimirim, Paracambí e Nova Iguaçu, distantes em média $50 \mathrm{~km}$ da capital. Todas estas áreas de coleta fazem parte ou circundam unidades de conservação, tais como o Parque Estadual da Pedra Branca, Parque Nacional da Tijuca, Parque Natural Municipal da Serra do Mendanha e Reserva Biológica do Tinguá. São ambientes que sofreram intensas explorações no passado, e que atualmente vêm sendo protegidos por dispositivos legais, que na prática não conseguem controlar o impacto do crescimento da malha urbana que os rodeiam. Os desmatamentos para estabelecimento de pastagens e culturas, o crescente processo de urbanização e favelização de centros urbanos, o comércio ilegal de espécies da fauna, a retirada da madeira e a introdução de espécies exóticas são elementos de contínua agressão, comuns a todos os remanescentes florestais do Estado (Rocha et al. 2003). Assim sendo, a apropriação deste espaço pela população circunvizinha se dá, entre outras formas, pelas atividades extrativistas (coleta de ervas e de plantas ornamentais, caça clandestina e cultivos diversos). Para Reis et al. (2002), parte considerável das unidades de conservação dos estados do Sul e Sudeste funciona como principal fonte de extração de recursos florestais, especialmente não madeireiros.

Assim como foi detectado por Williams et al. (2000) na África do Sul, percebeu-se que, com raras exceções, feirantes e mateiros ignoram o impacto do excesso de coletas sobre a diversidade regional das espécies. Neste sentido Almeida \& Albuquerque (2002) demonstram preocupação com o comércio de biorecursos e com a forte pressão extrativista em populações naturais e alertam para o fato de que as espécies comercializadas na feira de Caruaru vêm sofrendo técnicas de coleta agressivas visto ser a casca a principal parte da planta comercializada. Outros autores também destacam como relevantes a pressão de extração de plantas medicinais na Mata Atlântica (Reis et al. 2000; Montanari Junior 2002).

Pavan-Fruehauf (2000), em seu trabalho de manejo sustentado de plantas medicinais no Vale do Ribeira (São Paulo), lembra a ilegalidade do extrativismo devido à inexistência de estudos técnicocientíficos que possibilitem aos agentes dos órgãos governamentais o fornecimento de licenças para o extrativismo sustentado. Esta autora cita o decreto federal n. 750/93, que dispõe sobre corte, exploração e supressão de vegetação em áreas de domínio da Mata Atlântica, o qual prevê que a exploração seletiva de determinadas espécies nativas poderá ser efetuada desde que, além de outros requisitos, esteja baseada na elaboração de projetos fundamentados, entre outros aspectos, em estudos prévios de estoques e garantia de capacidade de manutenção da espécie.

Possivelmente a demanda decorrente da extração de plantas destinadas ao uso medicinal, mas principalmente aquelas de uso religioso, contribuam para uma maior pressão sobre as espécies nativas da Mata Atlântica no Rio de Janeiro. Desta forma, o envolvimento e parceria de mateiros, erveiros, pesquisadores e gestores do meio ambiente poderia apontar alternativas de extração racional, cultivo e produção de espécies utilizadas para fins religiosos. A presença do poder público é fundamental no sentido de disciplinar as coletas e também para oferecer alternativas cientificamente embasadas, principalmente para as populações da periferia da cidade do Rio de Janeiro, também no que se refere à utilização de plantas medicinais.

\section{Agradecimentos}

À FAPERJ, pela concessão de bolsa de Iniciação Científica à primeira autora; aos feirantes e mateiros que inúmeras vezes necessitaram interromper suas atividades, em especial à Sra. Rosana e Sr. Ademir, pela disponibilidade e boa vontade no oferecimento e troca de informações; à Maria Mercedes Teixeira da Rosa, Helena Regina Pinto Lima, Viviane Stern da Fonseca-Kruel e Ariane Luna Peixoto, pelas críticas e sugestões; aos taxonomistas do Departamento de Botânica da UFRRJ e Pesquisadores do Instituto de Pesquisas Jardim Botânico do Rio de Janeiro que auxiliaram na identificação do material botânico; aos revisores anônimos, pelas críticas e sugestões.

\section{Referências bibliográficas}

Alexiades, N.N. 1996. Select guidelines for ethnobotanical research: a field manual. New York, The New York Botanical Garden Press.

Almeida, C.F.C.B.R. \& Albuquerque, U.P. 2002. Uso e Conservação de plantas medicinais no estado de Pernambuco (Nordeste do Brasil): um estudo de caso. Interciência 27(6): 276-285.

Amorozo, M.C.M. 2002. Uso e diversidade de plantas medicinais em Santo Antonio do Leverger, MT, Brasil. Acta Botanica Brasilica 16(2): 189-203. 
Bennett, B.C. \& Prance, G.T. 2000. Introduced Plants in the Indigenous Pharmacopoeia of Northern South America. Economic Botany 54(1): 90-102.

Camargo, M.T.L.A. 1998. Plantas medicinais e de rituais afro-brasileiros II: estudo etnofarmacobotânico. São Paulo, Editora Ícone.

César, R.B. \& Oliveira, R.R. 1992. A Floresta da Tijuca e a cidade do Rio de Janeiro. Rio de Janeiro, Editora Nova Fronteira.

Cunningham, A.B. 1998. Plantas medicinales africanas. Orientaciones prioritarias em la intersección entre protección de la naturaleza y atención médica primaria. Documentos de trabajo de Pueblos y Plantas. v.1. Paris, UNESCO.

Falcão, V.J.A. 2002. Ewé, Ewé Ossain - Um estudo sobre os erveiros e erveiras do Mercado de Madureira: uma experiência do sagrado. Rio de Janeiro, Barroso Edições.

Farnsworth, N.R. 1997. Testando plantas para novos remédios. Pp. 107-125. In: E.O. Wilson (ed.). Biodiversidade. Rio de Janeiro, Editora Nova Fronteira.

Hersch-Martínez, P. 1995. Commercialization of Wild Medicinal Plants from Southwest Puebla, Mexico. Economic Botany 49(2): 197-206.

Lima, H.C.; Lewis, G.P. \& Bueno, E. 2002. Pau-brasil: uma biografia. Pp. 39-76. In: E. Bueno (ed.). Pau-brasil. São Paulo, Axis Mundi Editora.

Martin, G.M. 1955. Ethnobotany: a methods manual. 'People and Plants" Conservation Manuals. London, Chapman Hall.

Montanari Junior, I. 2002. Exploração econômica de plantas medicinais da Mata Atlântica. Pp. 35-54. In: L.L. Simões \& C.F. Lino (orgs.). Sustentável Mata Atlântica: a exploração de seus recursos florestais. São Paulo, Editora Senac.
Nodari, R.O. \& Guerra, M.P. 1999. Biodiversidade: aspectos biológicos, geográficos, legais e éticos. Pp. 11-24. In: C.M.O. Simõeset al. (eds.). Farmacognosia da planta ao medicamento. Porto Alegre, Editoras UFRGS/ UFSC.

Parente, C.E.T. \& Rosa, M.M.T. 2001. Plantas comercializadas como medicinais no Município de Barra do Piraí, RJ. Rodriguésia 52(80): 47-59.

Pavan-Fruehauf, S. 2000. Plantas medicinais de Mata Atlântica: manejo sustentado e amostragem. São Paulo, Editora Annablume.

Reis, M.S.; Mariot, A. \& Di Stasi, L.C. 2000. Manejo de Populações Naturais De Plantas Medicinais na Floresta Atlântica. Pp. 95-102. In: A.C. Diegues \& V.M. Viana (orgs.). Comunidades tradicionais e manejo dos recursos da Mata Atlântica. São Paulo, NUPAUB/ LASTROP.

Rocha, C.F.D.; Bergallo, H.G.; Alves, M.A.S. \& Sluys, M.V. 2003. A biodiversidade nos grandes remanescentes florestais do Estado do Rio de Janeiro e nas restingas da Mata Atlântica. São Carlos, Editora RiMa.

Sánchez, R.A.O. \& Valverde, R. 2000. Manual de cultivo y conservación de plantas medicinales. San José, R.A. Ocampo Editora

Trindade, O.J.S.; Bandeira, F.B.; Rêgo, J.C.; Sobrinho, J.L.; Pacheco, L.M. \& Barreto, M.M. 2000. Farmácia e Cosmologia: A Etnobotânica do Candomblé na Bahia. Etnoecológica 4(6): 11-32.

Ugent, D. 2000. Medicine, myths and magic the folk healers of a mexican market. Economic Botany 54(4): 427-448.

Williams, V.L.; Balkwill, K. \& Witkowski. E.T.F. 2000. Unraveling the commercial market for medicinal plants and plant parts on the Witwatersrand, South Africa. Economic Botany 54(3): 310-327. 